\title{
The early application of the calculus to the inverse square force problem
}

\author{
M. Nauenberg
}

Received: 10 December 2009 / Published online: 3 February 2010

(C) The Author(s) 2010. This article is published with open access at Springerlink.com

\begin{abstract}
The translation of Newton's geometrical Propositions in the Principia into the language of the differential calculus in the form developed by Leibniz and his followers has been the subject of many scholarly articles and books. One of the most vexing problems in this translation concerns the transition from the discrete polygonal orbits and force impulses in Prop. 1 to the continuous orbits and forces in Prop. 6. Newton justified this transition by lemma 1 on prime and ultimate ratios which was a concrete formulation of a limit, but it took another century before this concept was established on a rigorous mathematical basis. This difficulty was mirrored in the newly developed calculus which dealt with differentials that vanish in this limit, and therefore were considered to be fictional quantities by some mathematicians. Despite these problems, early practitioners of the differential calculus like Jacob Hermann, Pierre Varignon, and Johann Bernoulli succeeded without apparent difficulties in applying the differential calculus to the solution of the fundamental problem of orbital motion under the action of inverse square central forces. By following their calculations and describing some essential details that have been ignored in the past, I clarify the reason why the lack of rigor in establishing the continuum limit was not a practical problem.
\end{abstract}

\section{Introduction}

At the beginning of the eighteenth century, the differential calculus in the form developed by Leibniz was applied by his most prominent followers, Jacob Hermann, Pierre Varignon, and Johann Bernoulli, to the fundamental problem of planetary motion- to

Communicated by Niccolò Guicciardini.

M. Nauenberg $(\varangle)$

Physics Department, University of California Santa Cruz, Santa Cruz, CA, USA

e-mail: michael@physics.ucsc.edu 
describe the orbit of planets under the assumption that their motion is determined by an attractive force towards the Sun that varies inversely with the square of the distance. In 1679, Robert Hooke (Nauenberg 2005) wrote to Isaac Newton asking him

... and particularly if you will let me know your thoughts of that of compounding the celestiall motions of the planetts of a direct motion by the tangent $\&$ an attractive motion towards the centrall body (Newton 1960)

In the Principia, Newton took a great step forward by extending the application of Euclidean geometry to the concept of force impulses which he represented by line segments that in a limit have vanishing small magnitude,${ }^{1}$ leading to the emergence of a finite and continuous force. Such evanescent quantities appeared already in Greek geometry, in the method of exhaustion which, for example, was applied by Archimedes to obtain a rigorous relations between the circumference and the area of a circle, as well as bounds for these quantities that he calculated algebraically. ${ }^{2}$

Shortly after the publication of the Principia in 1687, Leibniz applied Prop. 1 to derive the now well-known differential equation of motion for central forces in polar coordinates. Then he gave an analytic proof that for elliptical orbital motion satisfying Kepler's area law, the resulting force depends inversely on the square of the distance from the center, ${ }^{3}$ in accordance with Newton's geometric proof in Prop. 11. Leibniz stimulated Johann Bernoulli and Pierre Varignon to apply the differential calculus to the solution of propositions in the Principia, and Jacob Hermann (1710a, 1710b $)^{4}$ used Cartesian coordinates instead of Leibniz's polar coordinates to show that under the action of inverse square forces, the orbit is a conic section. In Prop. 1, Newton described the orbit for a general central force by a polygon, see Fig. 1, which Hermann interpreted to be a sequence of chords of a continuous curve, see Fig. 2. He represented the Cartesian components of these cords by first-order differentials, and found that the components of Newton's line segments for the force impulses in Prop. 1, introduced geometrically in the Principia, were second-order differentials. ${ }^{5}$ Given

1 In the seventeenth century, the concept of a differential or infinitesimal quantity was not well defined. Newton observed that

Fermat in his method de maximis et minimis \& Gregory in his method of Tangents \& Newton (in his method) of the first and last ratios use the letter $o$ to signify a quantity not infinitely but indefinitely small $\&$ in this methods of tangents uses the letters $a \& e$ to become infinitely little...(Newton 1968).

The most colorful characterization of infinitesimals was given by Bishop George Berkeley, who called them "the ghost of departed quantities" (Boyer 1989).

2 Archimedes showed that the circumference $c$ of a circle is proportional to its diameter $d$, and that the area is proportional to the square of the diameter. He also calculated lower and upper bounds for the ratio $c / d=\pi$, namely, $223 / 71<\pi<22 / 7$.

3 Leibniz claimed that when he carried out his derivation he had not seen the Principia but only a review that had appeared in the Acta Eruditorum. It has been shown, however, that Leibniz's had obtained his heavily annotate copy of the Principia before the publication of his work (Bertoloni Meli 1991).

${ }^{4}$ Hermann had been a student of Jacob Bernoulli in Basel where he learned the calculus in Leibniz's notation.

5 Whiteside claimed, however, that the sides of the polygons in Prop. 1 are second-order differentials (Newton 1974), but his incorrect analysis has been warmly endorsed by I. B. Cohen in his Guide to 
any dependence of the force impulse with the distance from the center of force, Hermann obtained an exact algebraic relation of this second-order differential in terms of quadratic terms of the first-order differentials which mirrored Newton's geometrical relation. For the case that the force impulses vary inversely with the square of the distance, he solved his algebraic relation between finite differentials approximately by applying the rules of the differential calculus developed by Newton and Leibniz. It will be shown that a detailed study of Hermann's work is an excellent introduction to the calculus of differentials and its application to the solution of dynamical problems as it was practiced in the early part of the eighteenth century.

In his response to Hermann, Bernoulli (1710) criticized his approach, claiming that it was inadequate (Guicciardini 1999), and then he presented a different analytic method to evaluate the orbit for general central forces that was based on Newton's Prop. 41 integral. In a subsequent treatment, he also gave an alternate differential method based on Prop. 6 which will be shown to be equivalent to Hermann's treatment, except that in contrast with Hermann's use of Cartesian coordinates, Bernoulli, like Leibniz before him, made use of polar coordinates. Guicciardini has remarked that Bernoulli's evaluation of the integral in Prop. 41 for the case of an inverse square force was "the outcome of a protracted and successful research on the application of Liebniz's calculus, most notably differential equations, to natural philosophy" (Guicciardini 2008). But in contrast to Herman, who based his method on the differential calculus, Bernoulli evaluated the integral in Prop. 41 for the case of inverse square forces by methods of algebra and geometry that were already familiar to mathematicians in the seventeenth century including Fermat, Huygens, Newton, and Pascal (Boyer 1989; Kline 1972).

In the next section, I discuss Herman's analysis in Cartesian coordinates in some detail, because it is the simplest and most straightforward application of the differential calculus to the solution of the problem of planetary motion formulated geometrically in Newton's Principia. A new result that emerges from this discussion is the connection of Hermann's first integral to Newton's geometrical construction in Prop. 17. In Sect. 2, I describe the corresponding derivation in polar coordinates by Leibniz's which apparently even Newton had some difficulties to understand. ${ }^{6}$ In Sect. 3, I discuss the analysis of Bernoulli and Varignon who also represented differentials in polar coordinates, but instead of treating the force by a sequence of discrete impulses, they based their treatment on a representation of a continuous force in terms of the radius of curvature of the orbit which had been obtained in differential form by Bernoulli. ${ }^{7}$ The method employed by Bernoulli and Varignon, was based on an expression for the force in terms of the radius of curve communicated by d'Moivre to Bernoulli in 1706. Newton, who had derived this expression earlier, included it in the 1713 edi-

\footnotetext{
Footnote 5 continued

Newton's Principia (Cohen 1999). Evidently, in the eighteenth century, Newton's limiting procedures were better understood than in our present time. A detail discussion of Prop. 1 is given in reference (Nauenberg 2003).

6 Newton claimed erroneously that Leibniz had made an error in handling second-order differentials in his equation.

7 Bernoulli's differential expression for the curvature first appeared in section 78 of L'Hospital textbook of the calculus Analyse des Infiniment Petits pour L'Intelligence des Lignes Courbes.
} 
Fig. 1 Newton's diagram for Prop. 1, Book 1 of the Principia

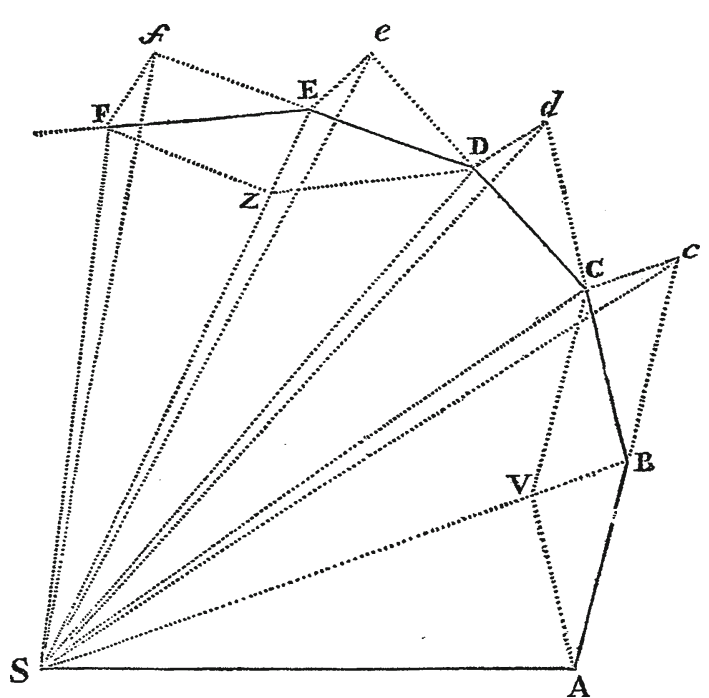

tion of the Principia, in a revised version of Prop. 6 that contained several additional corollaries. In Sect. 4, I discuss Bernoulli's solution based on Prop. 41, and describe a geometrical representation which he developed to present his result, followed by a proof that this representation corresponds to the algebraic equation for conic sections in Cartesian coordinates. ${ }^{8}$ Section 5 contains some conclusions followed by Appendix 1 on the early derivation of the fundamental theorem of the calculus, and Appendix 2 on a succint derivation with the aid of vector calculus of the results in the previous sections.

\section{Hermann's solution for the inverse square force problem}

Hermann's solution (1710a, 1710b) of the inverse problem of dynamics-given a central force to obtain all the orbits satisfying Kepler's area law-was based on the representation in Cartesian coordinates of Newton's geometrical construction in Prop. 1, Book 1 of the Principia, and on the application of the differential calculus. ${ }^{9}$ In Prop. 1, Newton gave a proof that under the action of a continuous central force, a body moves in a planar orbit, and the "areas describe by radii drawn to the center of force are proportional to the times". Newton started the proof of this proposition by assuming that the force consisted of a sequence of impulses in a plane directed towards a common center at equal time intervals. Then, in between impulses, the motion takes place along straight lines with constant velocity shown in his diagram, Fig. 1., as the polygon with sides $A B, B C$, etc. At each vertex of this polygon, Newton compounded

\footnotetext{
${ }^{8}$ For another discussion of the contributions of Leibniz, Bernoulli, Hermann, and Varignon to the inverse problem of central forces, see references Guicciardini (1999), Aiton (1962), and Aiton (1954).

9 The source of Hermann's diagram has been either neglected (Mazzone and Roero 1997; Bernoulli 2008) or errorneously assumed to be based on Prop. 6 of the Principia (Guicciardini 1999; Wilson 1994).
} 
Fig. 2 Herman's geometrical construction for an orbit for central forces

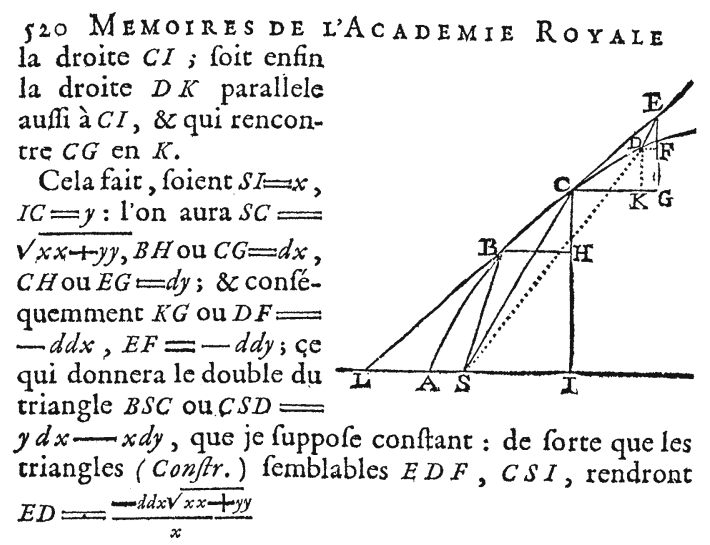

the previous velocity with the instantaneous change of velocity due to a force impulse directed towards the center at $S$. For example, in Fig. 1 the effect of an impulse at $B$ is described by a short line $C c$ parallel to $B S$, where $c$ is the position at the end of the time interval in the absence of the impulse, found by extending the line $A B$ by an equal length $B c$. Then Newton showed that the area of the triangles $A S B$ and $B S C$ are equal, and repeated this construction in sequence at each vertex of the polygon. ${ }^{10}$ He considered a continuous force to be the limiting case of a discrete sequence of such impulses as the time interval between these impulses becomes vanishingly small. Correspondingly, the areas of the triangles which are proportional to these time must also become vanishingly small. In order to indicate how such a limit could be approached geometrically, Newton referred to Lemma 3, Cor. 4 which presupposed the existence of a continuous curve such that the vertices of the polygon lie on the curve and the sides of the polygon are the chords of arcs of this curve. In this case, for each time interval, the magnitude of the displacements due to the force impulses are uniquely determined. In Lemma 11, Newton gave a proof that for such a limit to exist, the curve must have a finite radius of curvature.

In his diagram, Fig. 2, Hermann reproduced the first two vertices of the polygon in Prop. 1, but he turned the counter clockwise direction of rotation on this polygon around the center of force at $S$ into a clockwise rotation. To represent Newton's geometrical construction algebraically, Hermann introduced Cartesian coordinates, and obtained an expression for the force impulse $E D$ in terms of a second-order differential. It will be shown that Hermann's equations are exact algebraic relations between differentials, but that his two-step integration procedure to solve these equations for the case of an inverse square force involve approximations in that higher order differentials are neglected, in accordance with the rules of the differential calculus rules. These rules were formulated in the same form by Newton and by Leibniz. ${ }^{11}$

\footnotetext{
10 Since the time interval to travel along $B c$ and $B C$ are the same, it appears that the time interval to travel along $C c$ must vanish. To consider the continuum limit, however, Newton treated this time interval as a second-order differential.

11 By 1669, Newton had developed the differential form of the calculus, but his manuscript where circulated only privately by John Collins and were not published until 1711. Excerpts from the letter that Newton
} 
In his letter to Bernoulli, Hermann did not mention the origin of his geometrical representation of central forces, Fig. 2, but in a more detailed article in the Giornale de'Letterati d'Italia (Hermann 1710b), he acknowledged that it came from Newton's Prop. 1. By including in his diagram an orbital curve which was absent in Newton's diagram, Hermann showed explicitly how to obtain a continuous force as a limit of force impulses when the triangles associated with this construction are made vanishingly small. ${ }^{12}$ Hermann's method is equivalent to an earlier derivation by Leibniz, also based on Prop. $1,{ }^{13}$ who used polar coordinates in his derivation of a second-order differential equation for central forces. In his response letter to Hermann, Bernoulli also obtained Leibniz's differential equation in polar coordinates, but this derivation, which I will discuss below, was straightforward because it was based on a differential expression for the radius of curvature that he had obtained previously.

In Hermann's diagram, which consists of a segment of three consecutive vertices of the diagram associated with Prop. 1, the curve $A B C D$ represents an orbit, the center of force is at $S$, and the line $L E$ is obtained by extending the chord between two nearby points $B$ and $C$ on this curve on one side to $E$, where $C E=C B$, and on the other side to $L$ at the intersection of $L E$ with a horizontal line $L I$. The line $L I$ is the $x$-axis for a Cartesian coordinate system $x, y$, with the origin at $S$, which intersect the curve $A B C D$ at $A$. In this Cartesian representation, the coordinates of $C$ and $B$ are $x=S I, y=C I$, and $x-\mathrm{d} x, y-\mathrm{d} y$, respectively, where $\mathrm{d} x, \mathrm{~d} y$ are first-order differentials $\mathrm{d} x=B H$ and $\mathrm{d} y=C H$. The radial line $C S$ completes the triangle $C S B$, and its area in terms of the Cartesian coordinates can be shown to be $(1 / 2)(y \mathrm{~d} x-x \mathrm{~d} y)$. In Prop. 1, Newton gave a geometrical proof that under the action of a central force, with center at $S$, this area is proportional to the time interval $\mathrm{d} t$ for a body to move from $B$ to $C$. Following Newton's demonstration, in the absence of a force, a body would move, from $C$ to $E$ during a second equal time interval $\mathrm{d} t$. But when a force impulse towards $S$ occurs at $B$, the next location of the body is at $D$ on the curve $A B C D$, where its location is obtained by drawing from $E$ a line $D E$ that is parallel to $C S$ intersecting the curve. According to Prop. 1, the length of the segment $D E$ is a measure of the magnitude of the central force impulse

Footnote 11 continued

sent to Leibniz via Oldenburg and further material sent by Newton appeared in volume 2 of Wallis's Opera (1693) (Guicciardini 2009). Some of the principal concepts, however appeared in Lemmas in Sect. 1, Book 1, and in Lemma 2, Book 2 of the Principia. Leibniz published his first results in 1684 in the Acta Eruditorum, and he had contacts with the Johann and Jacob Bernoulli who promoted the early dissemination of the calculus in the Continent.

12 In Newton's words, "Now let the number of triangles be increased and their width decreased indefinitely, and their ultimate perimeter $A D F$ will (by lemma 3, corol. 4) be a curved line". In Hermann's diagram, Fig. 2, this limit "curved line" is $A B C D$, the center of force is at $S$, the central force impulse at $B$ is $D E$, and two of Newton's triangles of equal area are $B C S$ and $C D S$. In the diagram associated with Prop. 1, a limit curve is not drawn, but its existence is implied by Newton's reference to lemma 2, corol. 4. Indeed, such a curve is essential to specify Newton's geometrical construction when " the number of triangles be increased...indefinitely" (Nauenberg 2003). Although the necessity for assuming such a curve escaped the attention of contemporary commentators of Prop.1, it was recognized first by Leibniz and then by Herman in his diagram which represents the essential features of this proposition.

13 Leibniz claimed that he had obtained his result before he had seen a copy of the Principia, but his heavily annotated copy of Newton's has been found to pre-date his work (Bertoloni Meli 1991; Leibniz 1973), demonstrating a remarkable lack of candor on his part. 
at $B$, and the object of Hermann's diagram was to express this magnitude in terms of differentials. Representing the Cartesian coordinates of $D$ by $x+\mathrm{d} x^{\prime}, y+\mathrm{d} y^{\prime}$, where $\mathrm{d} x^{\prime}=C K$, and $\mathrm{d} y^{\prime}=D K$, the line segments $D F$ and $E F=$ representing the Cartesian components of $D E$ are determined by the second-order differentials $D F=-\mathrm{dd} x=\mathrm{d} x-\mathrm{d} x^{\prime}$, and $E F=-\mathrm{dd} y=\mathrm{d} y-\mathrm{d} y^{\prime} .{ }^{14}$ It is important to recognize that in contrast with the first-order differentials $\mathrm{d} x, \mathrm{~d} y$, where either one or a combination of the two can be chosen as an arbitrary small quantity, the subsequent differentials $\mathrm{d} x^{\prime}, \mathrm{d} y^{\prime}$ are both determined, when a curve $A B C D$ is assumed to be given. Hence, the second-order differentials $\mathrm{dd} x, \mathrm{dd} y$ also depend on the magnitude of these first-order differentials. ${ }^{15}$ Geometrically, this dependence is evident from Herman's diagram, Fig. 2, which shows that the position of $D$ on the curve $A B C D$ depends on the value chosen initially for $x$ and $\mathrm{d} x$. The object of the differential calculus is to find this curve given, the dependence of the central force $f$ on the distance $C S$, e.g., for an inverse square force, $f \propto 1 / C S^{2}$. By similarity of the triangles $E D F$ and CSI,

$$
E D=\operatorname{dd} x \frac{\sqrt{x^{2}+y^{2}}}{x}
$$

Assuming that the orbital curve $A B C D$ has a finite curvature, Newton gave a proof in Lemma 11 of Book 1 of the Principia that the magnitude of the force impulse $E D$ depends quadratically on the length $B C=\sqrt{\mathrm{d} x^{2}+\mathrm{d} y^{2}}$. Since the time interval $\mathrm{d} t$ is proportional to the area of the triangle $C B S=(1 / 2)|x \mathrm{~d} y-y \mathrm{~d} x|$, which depend linearly on $\mathrm{d} x$ and $\mathrm{d} y$, the ratio $E D / \mathrm{d} t^{2}$ has a finite limit when $\mathrm{d} x, \mathrm{~d} y$ become vanishingly small. In Prop. 6, Newton defined a continuous force $f$ by the relation (see Fig. 4)

$$
f \propto \frac{Q R}{(S P \times Q T)^{2}}
$$

where $Q R=(1 / 2) E D$ and $S P \times Q T$ corresponds to twice the area of the triangle $C B S$, in the limit that the triangle $C B S$ becomes vanishingly small. Substituting Hermann's second-order differential expression for $E D$, Eq. 1, in Newton's relation for the force, Eq. 2, leads to an expression for the force

$$
f \propto \frac{-\mathrm{dd} x \sqrt{x^{2}+y^{2}}}{x(y \mathrm{~d} x-x \mathrm{~d} y)^{2}},
$$

where the minus sign appears when $f$ is an attractive central force.

\footnotetext{
14 In section IV of the Analyse des Infinitment Petits, second-order differentials are defined as the difference between two consecutive first-order differentials, in accordance with Hermann's application.

15 At this stage, it should become clear why Hermann's analysis required three adjacent vertices from the polygon in Prop. 1, because a second-order differential like $\mathrm{dd} x$ is determined by the difference of two adjacent first-order differentials, $\mathrm{d} x$ and $\mathrm{d} x^{\prime}$.
} 
For an inverse square force, $f \propto 1 /\left(x^{2}+y^{2}\right)$, Herman then obtained an equation for the second-order differential $\mathrm{dd} x$ in the form

$$
\mathrm{dd} x=\frac{x(y \mathrm{~d} x-x \mathrm{~d} y)^{2}}{a\left(x^{2}+y^{2}\right)^{3 / 2}},
$$

where $a$ is a constant with the dimensions of length, introduced to convert Newton's proportionality relation for force, Eq. 2 into an equality. ${ }^{16}$ Such an algebraic relation between first- and second-order differentials, however, remains undetermined until a further condition is imposed on the first-order differential $\mathrm{d} x$ and $\mathrm{d} y$, because these differentials cannot be varied independently. Hermann's relation was obtained from Prop. 1 geometrical construction which leads to the constraint that the first-order differential $y \mathrm{~d} x-x \mathrm{~d} y$ is a constant, that is

$$
\mathrm{d}(y \mathrm{~d} x-x \mathrm{~d} y)=0 .
$$

This condition implements in differential form Kepler's area law which, in Prop. 1, Newton had shown to follow from central forces, and Hermann's differential relation, Eq. 4, was derived by applying this constraint. ${ }^{17}$ To integrate ${ }^{18}$ his differential relation, Hermann had to impose this condition which then becomes an additional second-order differential relation which he applied implicitly in his integration of Eq. 4.

In his treatment, Hermann neglected to consider an equivalent representation for $f$ obtained by substituting for $E D$ in Eq. 2 the expression obtained in terms of the second-order differential dd $y$,

$$
E D=\frac{\mathrm{dd} y}{y} \sqrt{x^{2}+y^{2}}
$$

When substituted in Newton's relation for the force, Eq. 2, this expression leads to the equivalent relation

$$
f \propto-\frac{\mathrm{dd} y \sqrt{x^{2}+y^{2}}}{y(y \mathrm{~d} x-x \mathrm{~d} y)^{2}},
$$

\footnotetext{
16 For an elliptic orbit, the constant $a$ is the latus rectum of the ellipse. Setting $y \mathrm{~d} x-x \mathrm{~d} y=l \mathrm{~d} t$ and $f=\mu /\left(x^{2}+y^{2}\right)$, where $l$ is the angular momentum, and $\mu$ is the strength of the inverse square force, leads to the relation $a=l^{2} / \mu$ that determines the value of $a$.

17 For central forces, this condition corresponds to the conservation of angular momentum $l$, which in Newton's fluxional calculus, as well as in modern calculus, is represented by $l=y \dot{x}-x \dot{y}$, and $\dot{l}=y \ddot{x}-x \ddot{y}=0$, The dot superscript corresponds to a derivative with respect to time.

18 In Leibniz's calculus, to integrate a relation between differentials meant to find a relation between differentials of a lower order which satisfies the requisite relation between the higher order differentials. By the fundamental theorem of the calculus, this operation corresponds to the calculation of the area under a given curve (see Appendix 1). It should be pointed out that without specifying the first-order differentials in the problem that are kept fixed, the concept of a second order or higher order differential is meaningless. In Hermann's calculations, the linear combination of differentials $y \mathrm{~d} x-x \mathrm{~d} y \propto \mathrm{d} t$ is kept fixed.
} 
and for an inverse square force, $f=1 /\left(x^{2}+y^{2}\right)$, leads to the second-order differential relation

$$
\mathrm{dd} y=-\frac{y(y \mathrm{~d} x-x \mathrm{~d} y)^{2}}{a\left(x^{2}+y^{2}\right)^{3 / 2}},
$$

which is analogous to Eq. 4, and must also be satisfied. By multiplying Eq. 4 by $y$ and Eq. 8 by $x$, one finds that the condition

$$
x \mathrm{dd} y-y \mathrm{dd} x=d(x \mathrm{~d} y-y \mathrm{~d} x)=0
$$

is then automatically satisfied when both Eqs. 4 and 8 are valid. Actually, this result is a straightforward proof, based on the application of the differential calculus, of Newton's theorem in Prop. 1 that "...equal areas are described during equal time intervals". But Hermann missed this simple proof, and it took another 6 years before he came up with a different and rather convoluted proof (Guicciardini 1999) that was based on the expression for the force in terms of the radius of curvature of the orbit. This alternate expression for the force appeared for the first time in the second edition of the Principia, and it was derived by Bernoulli who in turn had learned it from a private communication by De Moivre (Guicciardini 1999)

For the first integral of his second-order differential relation, Eq.4, Hermann set $(y \mathrm{~d} x-x \mathrm{~d} y)$ equal to a constant and obtained

$$
\mathrm{d} x=\frac{y(y \mathrm{~d} x-x \mathrm{~d} y)}{a \sqrt{x^{2}+y^{2}}} .
$$

But to establish this result, an essential approximation was to neglect all higher order terms in the differentials $\mathrm{d} x$, and $\mathrm{d} y$ that appear in the calculation of the differential on the right hand side of his relation. It is instructive to examine this step in some detail, because it illustrates the treatment of differentials in the calculus of Newton and Leibniz, and the nature of the approximations that are made at each step in its implementation.

The differential of the product of two quantities $u, v$ is, by definition,

$$
\mathrm{d}(u v)=(u+\mathrm{d} u)(v+\mathrm{d} v)-u v=u \mathrm{~d} v+v \mathrm{~d} u+\mathrm{d} u \mathrm{~d} v,
$$

but the assumption that $d u$ and $d v$ are small quantities which ultimately vanish, implies that in this limit the product $\mathrm{d} u \mathrm{~d} v$ can be neglected compared to the terms linear in $\mathrm{d} u$ and $\mathrm{d} v$, that is

$$
\mathrm{d}(u v) \approx u \mathrm{~d} v+v \mathrm{~d} u
$$

I have introduced here the sign $\approx$ to indicate that this relation is an approximation. ${ }^{19}$ It is the basis of the differential calculus of Newton and Leibniz, and appears

19 For finite values of $\mathrm{d} u$ and $\mathrm{d} v$, the common use of the equality sign, which unfortunately is standard form in the literature on this subject, leads often to confusion. 
prominently as Prop. II in L'Hospital 1696 book, Analyse des Infinitiment Petits, the first text-book on this new calculus. The justification given for neglecting $\mathrm{d} u \mathrm{~d} v$ is that

$\ldots \mathrm{d} u \mathrm{~d} v$ is a quantity infinitesimally small compared to the other terms $u \mathrm{~d} v$, and $v \mathrm{~d} u$; because if one divides, for example, $u \mathrm{~d} v$ and $\mathrm{d} u \mathrm{~d} v$ by $\mathrm{d} v$, one finds on the one hand $u$, and on the other $d u$ that is its differential, and consequently it is infinitesimally smaller than it. ${ }^{20}$

For Hermann's problem, let $u=y, v=1 / \sqrt{x^{2}+y^{2}}$, then the next step is to obtain an approximation for the differential $\mathrm{d} v$. Setting $z=1 / v$, and applying repeatedly the basic calculus rule, Eq. 12 , one finds $\mathrm{d} z^{2} \approx 2 z \mathrm{~d} z \approx 2(x \mathrm{~d} x+y \mathrm{~d} y)$ or $\mathrm{d} z \approx$ $v(x \mathrm{~d} x+y \mathrm{~d} y)$, and since $\mathrm{d}(z v)=0, \mathrm{~d} v \approx-v^{2} \mathrm{~d} z \approx-v^{3}(x \mathrm{~d} x+y \mathrm{~d} y)$. Hence

$$
\begin{aligned}
& u \mathrm{~d} v \approx-y \frac{(x \mathrm{~d} x+y \mathrm{~d} y)}{\left(x^{2}+y^{2}\right)^{3 / 2}}, \\
& v \mathrm{~d} u=\frac{\mathrm{d} y\left(x^{2}+y^{2}\right)}{\left(x^{2}+y^{2}\right)^{3 / 2}},
\end{aligned}
$$

and finally

$$
\mathrm{d}(u v)=\mathrm{d}\left(\frac{y}{\sqrt{x^{2}+y^{2}}}\right) \approx \frac{x(x \mathrm{~d} y-y \mathrm{~d} x)}{\left(x^{2}+y^{2}\right)^{3 / 2}}
$$

which verifies that Eq. 10 is the first integral of Eq. 4. Actually, the differential $\mathrm{d} v$ is a power series expansion, which according to Newton's generalized binomial theorem, consisted of an infinite number of terms of higher powers in $\mathrm{d} x$ and $\mathrm{d} y$. But a proof that the infinite sum of all these neglected terms is of higher order and therefore can be neglected, was not available. Hence, for a finite value of $\mathrm{d} x, \mathrm{~d}(u v) \mathrm{d} t \approx a \mathrm{dd} x$, and for finite differentials Eq. 10 satisfies Eq. 4 only approximately. But in accordance with the fundamental theorem of calculus, in the limit that $\mathrm{d} x$ becomes vanishingly small, ${ }^{21}$ Eq. 10 is an exact integral of Eq. 4.

In a similar way, Herman found that the integral of Eq. 10 is

$$
\frac{a}{x}=\frac{\sqrt{x^{2}+y^{2}}}{x} \pm c
$$

\footnotetext{
$20 \ldots \mathrm{d} u \mathrm{~d} v$ est une quantité infiniment petit par rapport aux autres terms $u \mathrm{~d} v \& v \mathrm{~d} u$; car si l'on divise, par example, $u \mathrm{~d} v \& \mathrm{~d} u \mathrm{~d} v$ par $\mathrm{d} v$, on trouve d'une part $u, \&$ de l'autre d $u$ qui en est la différence, \& par conséquent infinitment moindre qu'elle.

21 Regarding the approximations in relations involving finite differentials, Leibniz commented that

at the same time one has to consider that these ordinary incomparables themselves are by no means fixed or determined; they can be taken as small as one wishes in our geometrical arguments. Thus they are effectively the same as rigorous, infinitely small quantities, for if an opponent would deny our assertion, it follows from our calculus that the error will be less than any error which he will be able to assign, for it is in our power to take the incomparably small small enough for that, as one can always take a quantity as small as one wishes (Bos 1973).
} 
where $c$ is a constant. ${ }^{22}$ Again, this result can be verified by taking differentials on both sides of this relation, applying the Kepler area law condition (conservation of angular momentum), Eq. 9, and keeping only terms that appear in the resulting series expansion that are first order in the differentials $\mathrm{d} x$ and $\mathrm{d} y$. This relation is the algebraic equation for a conic section. ${ }^{23}$ with eccentricity $c$; a parabola for $c=1$, an ellipse for $c<1$, and a hyperbola for $c>1$. For an ellipse, the constant $a$ is the latus rectum, and $a /\left(1-c^{2}\right)$ is the major axis, providing the role of unit of length to the constant $a$ introduced by Hermann. Hermann's solutions constrain the axis of the conic to lie along an arbritraly chosen horizontal, but this restriction is invalid for arbitrary initial values of position and velocity. But by including in the first integral, the missing constant of integration $e(y x-x \mathrm{~d} y)$ pointed out by Bernoulli, one obtains the solution for a general conic section

$$
a=\sqrt{x^{2}+y^{2}}+c x+e y
$$

It is instructive to apply the differential calculus to obtain the solution of the direct problem in dynamics - given a planar orbit satisfying Kepler' area law, to obtain the radial dependence of the central force. In Props. 11-13, Newton gave a geometrical proof that for orbits that are conic section, the force depends inversely with the distance from a center. Starting with the Cartesian coordinate representation of a conic section given in Eq. 16, and taking his differential steps in reverse order by applying repeatedly the chain rule to this representation, leads to the expressions for the first- and second-order differential $\mathrm{d} x$, Eq. 10 , and $\mathrm{dd} x$, Eq. 4 , provided that the area $y \mathrm{~d} x-x \mathrm{~d} y$ of the triangle $B S C$ is kept constant. ${ }^{24}$ In Cartesian coordinates, Eq. 3 corresponds to Newton's definition for a central force $f$ given in Prop. 1 and Prop. 6, and substituting for $\mathrm{dd} x$ in this equation the relation given by Eq. 4 yields for the force the inverse square radial dependence, $f \propto 1 /\left(x^{2}+y^{2}\right)$.

\footnotetext{
22 The \pm sign corresponds to the two possible choices for the foci of a conic section as the center of the inverse square force.

23 In his evaluation, Herman introduced a spurious constant $b$, describing his result by the value of a constant $c / b$, “...qui est une équation aux trois Sections Conique; savoir à la Parabole si $b=c$, à 1 'Ellipse si $b>c, \&$ à 1 'dHyperbole si $b<c$ ".

24 The first-order differential of the algebraic equation for a conic section, Eq. 16, including an additional term $e y$, where $e$ was a constant pointed out by Bernoulli is
}

$$
\mathrm{d} a=\frac{x \mathrm{~d} x+y \mathrm{~d} y}{\sqrt{x^{2}+y^{2}}}+c \mathrm{~d} x+e \mathrm{~d} y .
$$

and since $a$ is a constant $\mathrm{d} a=0$ Setting $y \mathrm{~d} x-x \mathrm{~d} y=\mathrm{d} t$, where $\mathrm{d} t$ is treated as a constant when taken a differential of this relation, one obtains an equation for $\mathrm{d} x$ by substituting $\mathrm{d} y=(1 / x)(y \mathrm{~d} x-d t)$ in this equation,

$$
\left(\sqrt{x^{2}+y^{2}}+c x+e y\right) \mathrm{d} x=\frac{y \mathrm{~d} t}{\sqrt{x^{2}+y^{2}}}+e \mathrm{~d} t .
$$

But for the conic section, Eq. 16, the coefficient of $\mathrm{d} x$ is equal to $a$, which shows that this equation corresponds to Hermann's first integral, Eq. 10 including a missing constant of integration. 
Bernoulli responded to Hermann with a harsh criticism of his work Bernoulli (1710), remarking that Hermann had not provided any direct procedure to obtain the first integral, Eq. 10, of his second-order differential equation, Eq. 4 and that he had obtained his result because he already knew the answer - that his second integral was a conic section, Eq. 16. Actually in practice, integration of a nonlinear differential equation by an application of the fundamental theorem of the calculus usually involves an educated guess of the solution, followed by the verification that its derivative satisfies the differential equation. ${ }^{25}$ Bernoulli also pointed out that in the first integration Hermann had neglected to include a constant term. This term has to be a first-order differential, and since $y \mathrm{~d} x-x \mathrm{~d} y$ is treated as a constant, it has the unique form $e(y \mathrm{~d} x-x \mathrm{~d} y)$, where $e$ is an additional constant of integration. For this reason, Bernoulli stated that "one might be left in doubt that there is another kind of curve, other than the conic sections, that satisfies your problem", claiming that Herman had failed to prove that conic sections were a unique solution of his differential relation. Then Bernoulli showed that his constant leads to an additional term of the form $e y / x$ on the right hand side of Herman's second integral, because

$$
\mathrm{d}(y / x) \approx-\frac{1}{x^{2}}(y \mathrm{~d} x-x \mathrm{~d} y)
$$

It can be verified that with this additional term, Eq. 16 leads to the algebraic equation for a conic section with its major axis rotated relative to the $x$-axis by an angle $\omega=\operatorname{atan}(e / c)$. Ironically, Bernoulli's own proof that the solution he obtained from an application of Prop. 41 in Newton's Principia satisfied the algebraic equation for a conic section also was restricted to the special case that $e=0$, as will be shown in the next section.

Setting $\mathrm{d} t=(1 / l)(y \mathrm{~d} x-x \mathrm{~d} y)$, where $l$ is a constant correspond to the angular momentum, indicates that Herman's first integral, Eq. 10, with the additional constant $e$ introduced by Bernoulli, can be written in the alternate form

$$
e=\frac{a}{l} v_{x}-\frac{y}{\sqrt{x^{2}+y^{2}}}
$$

where $v_{x}=\mathrm{d} x / \mathrm{d} t$ is the $x$ component of the velocity $v$. If Herman had also solved for $v_{y}=\mathrm{d} y / \mathrm{d} t$, where $v_{y}=(y / x) v_{x}-l / x$, according to Kepler's area law, he would have found that

$$
c=-\frac{a}{l} v_{y}-\frac{x}{\sqrt{x^{2}+y^{2}}},
$$

Hence, given the position $x, y$ relative to the center of force, and the velocity $v_{x}, v_{y}$ at some point along the orbit, called the initial conditions, the angular momentum constant $l=y v_{x}-x v_{y}$ is fixed and all the parameters of the conic section are deter-

\footnotetext{
25 For this purpose, both Newton and Leibniz created extensive tables of derivates of elaborate functions, and ever since such tables have become a standard aid for the analytic evaluation of difficult integrals.
} 
mined in units of Hermann's length parameter $a$. In this form, Herman's result can be recognized as the components of a vector

$$
\vec{\epsilon}=\frac{\vec{v} \times \vec{l}}{\mu}-\frac{\vec{r}}{r}
$$

where $\vec{l}$ is the vector angular momentum taken normal to the plane of the orbit along the negative $z$ axis (Herman's diagram corresponds to clockwise rotation around the center of force $S$ ), and $\mu=a / l^{2}$ is a constant that determines the magnitude of the inverse square force, $f=\mu /\left(x^{2}+y^{2}\right)$. This vector $\vec{\epsilon}$ is a constant of the motion directed along the axis of conic section with a magnitude corresponding to its eccentricity $\epsilon$, where $\epsilon_{x}=c$, and $\epsilon_{y}=e$. Ninety one years later, it was derived in a similar manner by Simon Pierre Laplace (1798) who apparently was unaware of Herman's result. Actually, this special invariant of the motion for inverse square forces had already been described in geometrical form by Newton in the Principia, Book 1, Prop. 17, but this connection has not been made in the past. In this way, Prop. 17 establishes the uniqueness of conic sections, a fact that was already recognized by Euler (1736), but still leads to misconceptions up to the present time. In the first edition of the Principia, Newton gave an argument for the uniqueness of conic sections in Cor. 1 to Prop. 13 which was considered to be insufficient and lead to disputes. But in the second edition, Newton added the remark that

For if the focus and the point of contact and the position of the tangent are given, a conic can be described that will have a given curvature at that point. But the curvature is given from the given centripetal force and velocity of the body, and two different orbits touching each other cannot be described with the same centripetal force and the velocity.

In the differential calculus, the uniqueness of the conic section solution for inverse square force is based on the fundamental theorem of the calculus (see Appendix 1).

\section{Leibniz differential equation for motion in polar coordinates}

In 1688, after studying the Principia, Leibniz's was able to apply his calculus to obtained the differential equation of motion for central forces in polar coordinates which appeared in his Tentamen de motuum coelestium causis (Bertoloni Meli 1991). Like Hermann's derivation, Leibniz's treatment was also based on Newton's Prop. 1. For clarity, in Fig. 3 taken from E. J. Aiton (1995), I show only the section of Leibniz's diagram relevant to his analysis, which is also similar to Hermann's diagram, Fig. 2. The curve $M_{1}, M_{2}, M_{3}$ represents the orbit of planet moving under the action of a force centered at the point indicated here by the symbol for the Sun (a circle with a small dot in the center which will be called $S$ here). Leibniz draws the chord $M_{1} M_{2}$ which he extends by an equal length to a point $L$, and from there draws the line segment $L M_{3}$, parallel to the radial line $M_{2} S$ that intersects the curve at $M_{3}$. In this way, given any two nearby points $M_{1}$ and $M_{2}$ on the curve, the location of the third point $M_{3}$ is determined. Hence, except for change in notation, Leibniz's construction 
Fig. 3 A section of Leibniz diagram

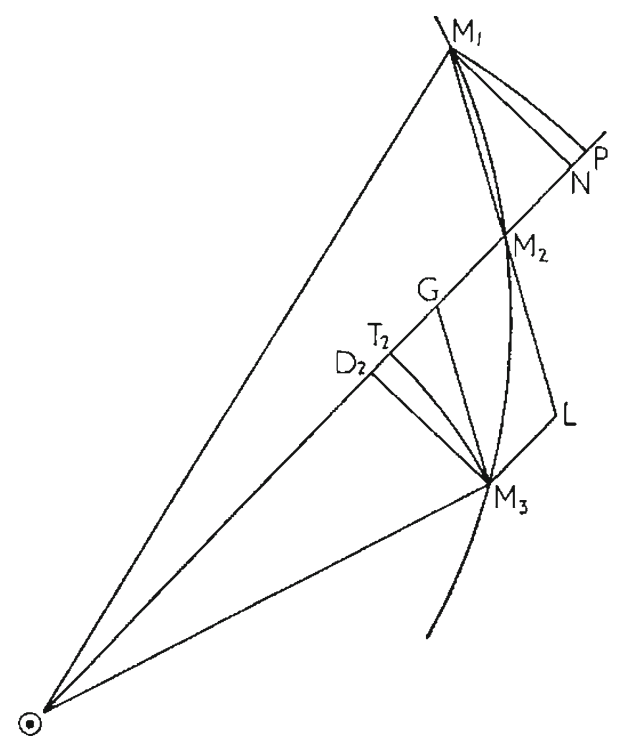

corresponds to Newton's construction in Prop. 1, shown in Fig. 1. The only difference is that Leibniz changed the sense of rotation in the Prop. 1 diagram to a clock-wise rotation, in the same manner that Hermann did later on in his diagram, Fig. 2.

The additional lines in Leibniz's diagrams are auxiliary lines to derive the necessary relations among the differentials. The lines $M_{1} N$ and $M_{3} D_{2}$ represent differential normals to the radial line $S M_{2}$ drawn from the vertices $M_{1}$ and $M_{3}$, respectively, and $M_{1} P$ and $M_{3} T_{2}$ are corresponding differential arcs centered at $S$. In addition, a line $M_{3} G$ is drawn parallel to $M_{2} L$. Then setting $S M_{1}=r-\mathrm{d} r, S M_{2}=r$ and $S M_{3}=r+\mathrm{d} r^{\prime}$,

$$
\mathrm{d} r=-\left(P N+N M_{2}\right)
$$

is the differential change in the radial coordinate from $M_{1}$ to $M_{2}$, and

$$
\mathrm{d} r^{\prime}=-M_{2} T_{2}=-\left(L M_{3}+G D_{2}-T_{2} D_{2}\right) .
$$

is the corresponding change from $M_{2}$ to $M_{3}$. By equality of the triangles $M_{1} M_{2} N$ and $M_{3} D_{2} G, N M_{2}=G D_{2}$, and $P N=D_{2} T_{2}$. Hence

$$
\mathrm{d} r^{\prime}=-\left(L M_{3}+N M_{2}-P N\right)
$$

and

$$
\mathrm{d} d r=\mathrm{d} r^{\prime}-\mathrm{d} r=-L M_{3}+2 P N
$$

where

$$
P N=\frac{\left(M_{1} N\right)^{2}}{2 S M_{2}} .
$$


to second order in $M_{1} N$. Since Leibniz followed Newton's geometric construction in Prop. 1, the triangles $M_{1} S M_{2}$ and $M_{2} S M_{3}$ have equal areas $\Delta=(1 / 2)\left(M_{1} N \times S M_{2}\right)$, and the elapsed time interval $\mathrm{d} t$ satisfies Kepler's area law $\mathrm{d} t=(1 / a) \Delta$. Here $a$ is a constant of proportionality introduced by Leibniz on dimensional grounds which we recognize as the angular momentum for unit mass. Setting $S M_{2}=r$, and $M_{1} N=$ $a \mathrm{~d} t / r$, one obtains

$$
P N=\frac{a^{2} \mathrm{~d} t^{2}}{2 r^{3}},
$$

Substituting in Eq. 27 this expression for $P N$ and $L M_{3}=f \mathrm{~d} t^{2}$, where $f$ is the central force, Leibniz obtained the now well-known differential equation of motion in polar coordinates,

$$
\mathrm{dd} r-\frac{a^{2} \mathrm{~d} t^{2}}{r^{3}}=-f \mathrm{~d} t^{2}
$$

Leibniz also demonstrated that when the orbital curve is an ellipse and the motion satisfies Kepler's area law, $\mathrm{d} \Delta=0$, the force $f$ is an inverse square force $f \propto 1 / r^{2}$. His proof, which he obtained by evaluating the second-order differential $\mathrm{dd} r$ from a first-order differential expression for the elliptical orbit (Aiton 1995), corresponds to Newton's geometric proof in Prop. 11.

It has been argued that Leibniz's representation of orbits by discrete polygons instead of continuous curves was just a matter of convenience (Aiton 1962; Bertoloni Meli 1991; Guicciardini 2008), but this is not the case. In Prop. 6, Newton represented a segment of the orbit by an arc, $P Q$, and the force by the deviation $Q R$ from a line $Z P R Y$ tangent at one end $P$ of the arc, Fig. 4 . In order to apply the differential calculus to this representation, however, an analytic expression for this tangent line is required, but this is an unknown quantity which cannot be expressed in terms of differentials from the geometric quantities given in Prop. 6. In fact, three nearby points of the orbit are required to obtain a unique circular arc, and then the tangent line at any point on this arc can also be obtained. Such a construction was pointed out to Leibniz by Pierre Varignon, and if the tangent line is placed midway on the arc, it leads to half as large a value for the force impulse obtained by Leibniz and by Hermann (Guicciardini 2008). This procedure is equivalent to Leibniz's original construction, Fig. $3 .^{26}$

Although Leibniz derived his analytic equation of motion directly from Newton's geometrical construction in Prop. 1, he gave it a completely different physical interpretation. Regarding the issue of how mathematics can mirror reality, it is of interest to discuss Leibniz's interpretation, because it illustrates ambiguities that can occur at the initial stages when a new theory is in the first stage of development. Following

${ }^{26}$ In the second edition of the Principia, Newton altered Prop. 6 to read

...the sagitta of the arc is understood to be drawn so as to bisect the chord and, when produced, to pass through the center of force...

This sagitta is the displacement associated with the force, which corresponds to Varignon's construction, but Newton did not alter the diagram, Fig. 4, associated with Prop. 6 accordingly. 
Fig. 4 Newton's diagram for Prop. 6, Book 1 of the Principia

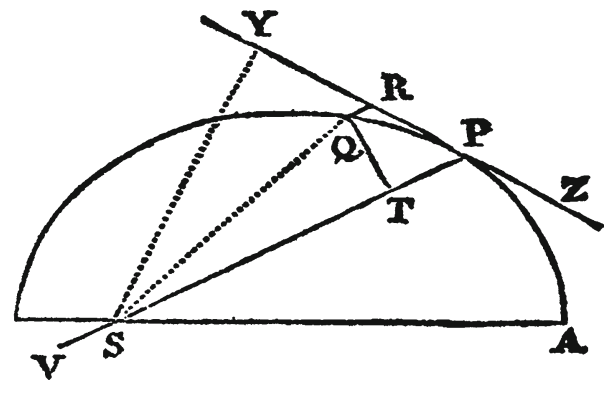

the Cartesian argument that planets rotate around the sun because they are carried by vortices of unseen particles or a fluid circulating the sun, Leibniz argued that Kepler's area law gave a mathematical description of these vortices, namely, that the circulation velocity of a vortex depends inversely with the distance from the sun. A consequence of Leibniz's interpretation is that planets experience an acceleration or force which, in accordance with Prop. 2 in the Principia ${ }^{27}$ is directed towards the sun, and is represented by $\mathrm{LM}_{3}$ in Leibniz's diagram, Fig. 3, but Leibniz did not give any justification that this force must be a central force. For circular motion, this central force which Leibniz re-named solicitation of gravity is balanced by an outward centrifugal conatus represented by the term $a^{2} \mathrm{~d} t^{2} / r^{3}$ in Leibniz's equation of motion, Eq. 30. Leibniz interpreted this term as a real force due to the Cartesian vortices acting on the planet, which depends on the parameter $a$. Since the rotational velocity of the planet is the same as that of the vortex, $a$ is also an intrinsic property of the planet corresponding to it angular momentum. Planets, however, do not rotate in circular orbits, as would be expected in this Cartesian model, but instead move in elliptical orbits crossing different vortices, and Leibniz explained the variation in radial distance, expressed by the second-order differential $\mathrm{dd} r$, by the difference between the solitication of gravity and the centrifugal connatus. But this radial acceleration is also a consequence of Prop. 1 , and it does not require the existence of vortices. In fact, such an explanation for the radial oscillations in the orbit was already given by Newton in a cryptic remark in his 1679 correspondence with Hooke, and in his 1681 correspondence with Crompton regarding a question of Flamsteed about the motion of comets near the sun (Nauenberg (1994)). The vortex interpretation had one advantage over Newton's theory, because it offered an explanation why the planets all rotated along the same direction. ${ }^{28}$ But this explanation had to be abandoned when comets were found to rotate around the sun in directions opposite to that of the planets. ${ }^{29}$

\footnotetext{
27 In Prop.2, Newton gives a proof that "every body...that describes areas around a point proportional to the times, is urged by a centripetal force toward the same point". But Newton's proof is based on the concept of inertial straight line motion in the absent of an external force, which Leibniz ignored.

28 The modern explanation is based on the theory for the origin of the planets from an initial dense gas of particles circulating the sun which holds also for numerous recently discovered extrasolar planetary systems.

29 It is interesting to compare the seventeenth century vortex theory based on unobserved particles with our current speculations of dark matter. This matter, which is also associated with unobserved particles, has been invoked to account for the peculiar rotational velocity of some stars that cannot be explained with our current theory of gravity by the amount of observable matter in galaxies.
} 


\section{Bernoulli's and Varignon's derivations of Prop. 39 and 41.}

In his response letter to Hermann (Bernoulli 1710), Bernoulli applied the differential calculus to derive Newton's expression for the energy conservation principle, Prop. 39 , and the integral for the orbital curve in polar coordinates, Prop. 41, for a general central force. A similar derivation was given by Varignon in the same issue of the Memoires of the Academy des Sciences where the letters of Hermann and Bernoulli appeared. Varignon's derivation differs from Bernoulli's only in the form that each one used for the central force. In 1706, Bernoulli learned the expression for a central force in terms of the radius of curvature $\rho$ of the orbit from a correspondence with De Moivre, although he did not acknowledge it (Guicciardini 2008). ${ }^{30}$ On the other hand, by following Newton's geometrical derivation of Prop. 39, Varignon obtained an expression for the force in terms of the second-order differential of the arc length of the orbit. Bernoulli gave an expression for $\rho$ in terms of first and second-order differentials in polar coordinates, without indicating how he obtained it. ${ }^{31}$ But his derivation of the equations of motion also follow directly from Herman's algebraic treatment of Prop. 1 by using polar coordinates $r, \theta$ instead of Cartesian coordinates $x, y$. It is instructive to follow these steps which are similar to Leibniz's original derivation of the differential equations of motion in polar coordinates discussed in the previous section. ${ }^{32}$

Turning to Bernoulli's notation, $r=x$ and $r \mathrm{~d} \theta=\mathrm{d} y$, Eq. 38 corresponds to Bernoulli's expression for the force, which he obtained by applying the relation for the central force $f$, in terms of the curvature of the orbit, that he had obtained from De Moivre,

$$
f=\frac{x}{p^{3}(2 \rho)}
$$

where $p=r \sin \psi=\mathrm{d} y / \sqrt{\mathrm{d} x^{2}+\mathrm{d} y^{2}}$, and $\rho$ is the radius of curvature of the orbit. For $\rho$, Bernoulli wrote the expression

$$
\rho=\frac{x\left(\mathrm{~d} x^{2}+\mathrm{d} y^{2}\right)^{3 / 2}}{\mathrm{~d} x^{2} \mathrm{~d} y+\mathrm{d} y^{3}+x \mathrm{~d} x \mathrm{dd} y-x \mathrm{~d} y \mathrm{dd} x},
$$

\footnotetext{
30 Newton had derived this relation earlier, but he did not include it in the first edition of the Principia (Nauenberg 1994). It appear in the second edition (1713) of the Principia in several new corollaries to a revised form of Prop. 6. In Lemma 11, Newton discussed the radius of curvature to justify the existence of the ratio, Eq. 2, that gives the magnitude of a central force in Prop. 6 in the limit that the square of the area of the triangle in this proposition becomes arbitrarily small.

31 In 1691, Bernoulli obtained a differential expression for the radius of curvature $\rho$ that impressed L'Hospital, who offered him a position as his private tutor in the differential calculus (Bos 1973). L'Hospital's celebrated Analyse des Infiniment Petits was based on Bernoulli's lectures, and he devoted an entire section entitled Usage des calcul des differences pour trouver les Dévelopées to Huygens's theory of evolutes based on Bernoulli's 15th lecture (Bernoulli 1914). Johann Bernoulli's older brother Jacob Bernoulli also derived and expression for $\rho$ by applying the calculus. The source for both Bernoulli brothers was the general expression for $\rho$ in Huygens's Horologium Oscillatorium (1673).

32 In Hermann's diagram, Fig. 2, set $S C=r, S B=r-\mathrm{d} r, S D=r+\mathrm{d} r^{\prime}$, and with vertex at $S$, set angle $B S D=\mathrm{d} \theta$ and angle $C S D=\mathrm{d} \theta$. Let $\psi$ be the angle $B C S$ with vertex at C. Then
} 
without indicating its origin, ${ }^{33}$ and restricting the variation of the differentials by the area law condition which in polar coordinates takes the form

$$
\mathrm{d}(x \mathrm{~d} y)=x \mathrm{dd} y+\mathrm{d} x \mathrm{~d} y=0
$$

Footnote 32 continued

$$
B C \sin (\psi)=(r-\mathrm{d} r) \mathrm{d} \theta
$$

and the conditions, $C D$ parallel to $B C$, leads to the relation

$$
C E \sin (\psi)=\left(r+\mathrm{d} r^{\prime}\right) \mathrm{d} \theta^{\prime}
$$

Since $C E=C D=B C$, by equating Eqs. 31 and 32, I obtain

$$
\mathrm{dd} \theta=\mathrm{d} \theta^{\prime}-\mathrm{d} \theta=\frac{2}{r} \mathrm{~d} r \mathrm{~d} \theta
$$

which relates the second-order differential $\mathrm{dd} \theta$ to the first-order differentials $\mathrm{d} r$ and $\mathrm{d} \theta$. To third order in the differentials $\mathrm{d} r$ and $\mathrm{d} \theta$, I find that

$$
B C^{2}=\mathrm{d} r^{2}+r(r-\mathrm{d} r) \mathrm{d} \theta^{2}
$$

and

$$
C E^{2}=\mathrm{d} r^{\prime 2}+r\left(r+\mathrm{d} r^{\prime}\right) \mathrm{d} \theta^{\prime 2}+2 E D \mathrm{~d} r
$$

and taking the difference between these two terms, I obtain

$$
C E^{2}-B C^{2}=2\left(\mathrm{~d} r \mathrm{dd} r+r^{2} \mathrm{~d} \theta \mathrm{dd} \theta+r \mathrm{~d} r \mathrm{~d} \theta^{2}+E D \mathrm{~d} r^{\prime}\right)
$$

Then, setting $C E=B C$, and substituting for $\mathrm{dd} \theta$ the expression given in Eq. 33, yields

$$
E D=r \mathrm{~d} \theta^{2}-\mathrm{dd} r
$$

This relation for the impulse $E D$ was first derived by Leibniz using an equivalent approach (Aiton 1962). In polar coordinate, the area of triangles $B S C$ an $C S E$, which are equal by construction, is $(1 / 2) r^{2} \mathrm{~d} \theta$ and according to Prop. 6, the force $f$ given by Eq. 2, is

$$
f \propto \frac{r \mathrm{~d} \theta^{2}-\mathrm{dd} r}{\left(r^{2} \mathrm{~d} \theta\right)^{2}}
$$

33 This expression for the radius of curvature $\rho$ can be obtained by substituting polar coordinates in an expression for $\rho$ in Cartesian coordinates obtained by Jacob Bernoulli. 
he obtained ${ }^{34}$

$$
\rho=\frac{x\left(\mathrm{~d} x^{2}+\mathrm{d} y^{2}\right)^{3 / 2}}{\left(\mathrm{~d} y^{3}-x \mathrm{~d} y \mathrm{dd} x\right)}
$$

Substituting this expression for $\rho$ in Eq. 39 yields. ${ }^{35}$

$$
\phi=\frac{\mathrm{d} y^{3}-x \mathrm{~d} y \mathrm{dd} x}{2 c^{3}}
$$

where $c=x \mathrm{~d} y$ Apart from a factor 2, this is the same expression for the force, Eq. 38, obtained directly from Hermann's diagram. Multiplying both sides of this equation by $\mathrm{d} x$, and applying Eq. 41 to substitute $\mathrm{d} x \mathrm{~d} y=-x \mathrm{dd} y$ on the right hand side yields

$$
\phi \mathrm{d} x=-\frac{\mathrm{d}\left(\mathrm{d} x^{2}+\mathrm{d} y^{2}\right)}{4 c^{2}},
$$

or equivalently

$$
\int \phi \mathrm{d} x=-\frac{\mathrm{d} x^{2}+\mathrm{d} y^{2}}{4 c^{2}} \pm n
$$

where $n$ is a constant of integration. Setting $c=\mathrm{d} t$, this relation can be recognized as a derivation via the differential calculus of the principle of conservation of energy $E$ for central forces, ${ }^{36}$ where $E=2 n$ that Newton had demonstrated by a geometrical

34 Since Hermann analysis satisfies Kepler's area law condition $\mathrm{d}(x \mathrm{~d} y)=0$, Bernoulli's restricted expression for $\rho$, can be obtained by finding the radius of a circle containing the points $B, C$ and $E$ in Hermann's diagram of Prop 1, Fig. 2. The center of this circle is located at a point $O$ where three lines of equal length drawn from $B, C$ and $E$ intersect. Hence the length $\rho$ of these lines is determined by the differential chords $B C$ and $C E$ and their relative orientation. Bernoulli's expression differs, however, from the conventional expression for $\rho$ in polar coordinates, first obtained in 1671 by Newton (Newton 1670-1673), which has the form

$$
\rho=\frac{\left(\mathrm{d} r^{2}+r^{2} \mathrm{~d} \theta^{2}\right)^{3 / 2}}{\mathrm{~d} \theta\left(r^{2} \mathrm{~d} \theta^{2}+2 \mathrm{~d} r^{2}-r \mathrm{~d}^{2} r\right)}
$$

because the second-order differential $\mathrm{d}^{2} r$ is obtained by assuming that $\mathrm{d} \theta$ remains constant.

35 Bernoulli's notation for force is $\phi$.

36 A modern version of Bernoulli's derivation starts with his differential equations in the form

$$
\ddot{r}-r d \theta^{2}=-\phi
$$

where $l=r^{2} \dot{\theta}$ is the constant angular momentum. Applying the identities

$$
\dot{r}\left(\ddot{r}-\frac{l^{2}}{r^{3}}\right)=\frac{1}{2} \frac{\mathrm{d}}{\mathrm{d} t}\left(\dot{r}^{2}+\frac{l^{2}}{r^{2}}\right),
$$


analysis in Prop. 39. Finally, substituting $\mathrm{d} y=c / x$, Bernoulli obtained

$$
\mathrm{d} y=\frac{\mathrm{d} x}{\sqrt{4 x^{2}\left(n-\int \phi \mathrm{d} x^{\prime}\right)-1}}
$$

which is a relation between first-order differentials corresponding to Newton's quadrature or integral in Prop. 41 given in terms of the differential $\mathrm{d} z=a \mathrm{~d} \theta=a \mathrm{~d} y / x$.

In an article that followed the exchange of letters between Hermann and Bernoulli, Pierre Varignon gave another derivation of Prop. 39 and 41 based on the differential calculus (Varignon 1710). Varignon's derivation follows Newton's original derivation in the Principia by starting with a representation in polar coordinates of Proposition 39 for the conservation of energy in the language of the calculus. For an attractive force, the component $f_{t}$ tangent to the orbit is

$$
f_{t}=-\mathrm{dd} s / \mathrm{d} t^{2}
$$

where $\mathrm{d} s$ is the differential arc length traversed during a differential interval of time $\mathrm{d} t$. For a central force $f, f_{t}=f \mathrm{~d} r / \mathrm{d} s$, which leads to Varignon's relation for $f$, apart from a missing minus sign,

$$
f=-\frac{\mathrm{d} s \mathrm{dd} s}{\mathrm{~d} r \mathrm{~d} t^{2}}
$$

Introducing polar coordinates $y, z$, where $y=r$ is the radial distance, $z$ is the polar angle in units of the arc of a circle with arbitrary radius $a$, and $\mathrm{d} x=y \mathrm{~d} z$. Then $y \mathrm{~d} x$ corresponds to the equal areas of the differential triangles in Prop. 1 and is proportional to the differential time interval $\mathrm{d} t$. Varignon failed to recognize that according to Prop. 1, this relation is a direct consequence of the assumption that the force $f$ is a central force, but he did consider as an example, the case that the second-order

Footnote 36 continued

and

$$
\dot{r} \phi=\frac{\mathrm{d}}{\mathrm{d} t}\left(\int^{r} \phi \mathrm{d} r\right)
$$

to the relation

$$
\dot{r}\left(\ddot{r}-\frac{l^{2}}{r^{3}}\right)+\dot{r} \phi=0
$$

then shows that

$$
\frac{1}{2}\left(\dot{r}^{2}+\frac{l^{2}}{r^{2}}\right)+\int \phi \mathrm{d} r=E
$$

where $E$ is a constant corresponding to the total energy. 
differential $\mathrm{dd} s$ is evaluated under the hypothesis that $y \mathrm{~d} x$ and $\mathrm{d} t$ are kept constant. Setting $\mathrm{d} s \mathrm{dd} s \approx(1 / 2) \mathrm{d}\left(\mathrm{d} s^{2}\right)$, leads to the first integral of Eq. 54

$$
2 \int f \mathrm{~d} y=-\frac{\mathrm{d} s^{2}}{\mathrm{~d} t^{2}}+n
$$

where $n$ is a constant. Since $\mathrm{d} s / \mathrm{d} t$ is the velocity, we recognize that $n=2 E$ where $E$ is the modern constant representing the conservation of energy principle.

The next step in Varignon's derivation was to express the differential arc length $\mathrm{d} s$ in polar coordinates, $\mathrm{d} s=\sqrt{\mathrm{d} x^{2}+\mathrm{d} y^{2}}$, and to set $\mathrm{d} t=y \mathrm{~d} x$. Substituting this expression in Eq. 55, and solving the resulting algebraic equation for $\mathrm{d} x$, he obtained

$$
\mathrm{d} x=\frac{\mathrm{d} y}{\sqrt{n y^{2}-2 y^{2} \int f \mathrm{~d} y-1}},
$$

Varignon's result corresponds to Newton's result in Prop. 41, and to Bernoulli's result, Eq. 52, except that Newton introduced a constant $Q=y \mathrm{~d} x / \mathrm{d} t$ corresponding to the angular momentum, which then leads to his relation

$$
\mathrm{d} x=\frac{Q \mathrm{~d} y}{\sqrt{n y^{2}-2 y^{2} \int f \mathrm{~d} y-Q^{2}}},
$$

except for the factor 2 in the denominator. But neither Varignon, nor Bernoulli or Hermann considered the integration of $\mathrm{d} t=(1 / Q) y \mathrm{~d} x$ to determine the time along the orbit.

\section{Bernoulli's integration of the quadrature in Prop. 41 for an inverse square force}

In his letter to Hermann, Bernoulli translated into Leibniz's notation Newton's description in Prop. 41 for an integral that determines the dependence of the polar angle of an orbit on the radial distance from the center of a general central force $f$ (Bernoulli 1710; Brackenridge 2003; Erlichson 1994). Afterwards, he also derived this integral from a differential expression for the force, as has been shown in the previous section. For an inverse square force, $f=a^{2} g / x^{2}$, where $x$ is the radial distance from the center of force. ${ }^{37} a$ is a constant with dimension of length, and $g$ is a constant with dimension of acceleration, Bernoulli obtained ${ }^{38}$

$$
\mathrm{d} z=\frac{a^{2} c \mathrm{~d} x}{x \sqrt{a b x^{2}+a^{2} g x-a^{2} c^{2}}},
$$

\footnotetext{
37 In conformance with the notation in Bernoulli's article, in this section, $x$ refers to the radial coordinate $r$ instead of the conventional cartesian coordinate $x$ for the horizontal axis.

38 The constant $a b=2 E$ where $E$ is the total energy, and the constant $a c=l$ where $l$ is the angular momentum. Bernoulli dropped a factor 2 which should multiply the constant $a^{2} g$.
} 
where $\mathrm{d} z=a \mathrm{~d} \theta$ is an infinitesimal arc of a circle with radius $a$. Bernoulli obtained this result by a direct translation of Newton's expression for this arc, given for an arbitrary central force in geometrical language, into the cartesian notation for differentials developed by Leibniz. ${ }^{39}$ In order to transform this expression into the "ordinary differential of the arc of a circle", Bernoulli's first step was to introduce the inverse transformation

$$
x=\frac{a^{2}}{y},
$$

to a variable $y$, which leads to the relation

$$
\mathrm{d} z=-\frac{c \mathrm{~d} y}{\sqrt{a b+g y-\left(c^{2} / a^{2}\right) y^{2}}} .
$$

Then shifting the origin of the coordinate $y$ by setting $y=\alpha-t$, where $\alpha$ is a constant and $t$ is a variable, Bernoulli determined $\alpha$ by the requirement that on substitution of this expression for $y$ in the argument of the square root in Eq. 60, the term linear in $t$ vanish. This condition gives $\alpha=a^{2} g / 2 c^{2}$, and in terms of the new variable $t$ he obtained

$$
x=\frac{a^{2}}{a^{2} g / 2 c^{2}-t},
$$

and

$$
\mathrm{d} \theta=\frac{\mathrm{d} z}{a}=\frac{\mathrm{d} t}{\sqrt{h^{2}-t^{2}}},
$$

where $h=(a / c) \sqrt{a b+a^{2} g^{2} / 4 c^{2}}$. At this point, Bernoulli recognized that while the left hand side of Eq. 62 is the differential angle for the polar angle $\theta=z / a$ of the orbit, the right hand side of this equation is the expression in cartesian coordinates for the differential angle associated with a circle of radius $h .^{40}$ In Cartesian coordinates $x, y$, the differential length of the arc is $\mathrm{d} s=\sqrt{\mathrm{d} x^{2}+\mathrm{d} y^{2}}$, and for a circle of radius $h=\sqrt{x^{2}+y^{2}}$,

$$
\mathrm{d} h=\frac{x \mathrm{~d} x+y \mathrm{~d} y}{h}=0 .
$$

Hence $\mathrm{d} y=-(x / y) \mathrm{d} x$, and substituting this relation for $\mathrm{d} y$ in $\mathrm{d} s$ gives $\mathrm{d} \theta=\mathrm{d} s / h=$ $\mathrm{d} x / \sqrt{h^{2}-x^{2}}$. On geometrial grounds, this identification had already been made by

\footnotetext{
39 The only additional step that Bernoulli had to undertake was the evaluation of the integral in Prop. 39 for the area bounded by the curve associated with an inverse square force, namely $\int \mathrm{d} x\left(1 / x^{2}\right)=-1 / x$. Such integrals, however, were already well known over half a century earlier from the work of Fermat (Kline 1972). Presently, this integral is called the potential for the inverse square force.

40 ...qui est une differentielle d' arc de cercle dont le rayon est $=h, \&$ son sinus $=t$ divise par son rayon.
} 
several mathematician in the seventeenth century. Hence, in modern notation ${ }^{41}, t=$ $h \sin \left(\theta-\theta_{o}\right)$, and Eq. 61 takes the form

$$
x=\frac{2 c^{2} / g}{1-\epsilon \sin \left(\theta-\theta_{o}\right)},
$$

which is the now familiar equation in polar coordinate for a conic section with eccentricity

$$
\epsilon=2 c^{2} h / a^{2} g=\sqrt{1+4 c^{2} b / a g^{2}} .
$$

In the tradition of seventeenth century mathematics, Bernoulli also gave a geometrical construction to describe this conic section, but his description has not received the attention is deserves.

Referring to Fig. 5, the axis for the cartesian coordinates $x, y$ are the inverted vertical line $R Q$, and the horizonal line $O Q$ respectively with origin at the intersection $Q$. The inverse relation between the variables $x$ and $y$ is expressed by the curve $V X Z$ which is a hyperbola determined by the proportion

$$
\frac{X Y}{O Z}=\frac{O Q}{Y Q} \text {. }
$$

where $x=X Y, y=Y Q$. The condition that for $y=a^{2} g / 2 c^{2}$ we have $x=2 c^{2} / g$ implies that $O Q=a^{2} g / 2 c^{2}$ and $O Z=2 c^{2} / g$, and then $O Z \cdot O Q=a^{2}$ The connection to the angular variable is obtained by shifting the origin of coordinates from $Q$ to the point $O$ along the horizontal $y$-axis, and constructing a circle $T S M$ centered at $O$, with radius

$$
O T=O M=h
$$

where $O T$ is along the horizontal and $O M$ along the vertical axis. Then $O Y=t$ and the condition

$$
O A=O Z=\frac{2 c^{2}}{g} .
$$

and $t=Y O=O Q-Y Q$. The intersection at $S$ of a straight line $O S$ with the circle $T S M$, drawn at an angle $\theta=S O M$ from the vertical line $O A$, determines $Y$ and $X$ by the intersections of a vertical line through $\mathrm{S}$ with the horizontal line $O Q$ and the hyperbola $V X Z$, respectively. Then extending the line $O S$ to $B$, such that $O B=X Y$ is the radial distance, and varying the angle $\theta=S O M$ with vertex at $O$, the path of $B$ describes geometrically the curve $A B C$ corresponding to orbit, where

\footnotetext{
41 Bernoulli did not use a notation for the ratio $t / h$ which corresponds to the trigonometric function sine of the angle $\theta$.
} 
Fig. 5 Bernoulli's geometrical construction for the orbit of a conic section

Soit donc ici (où les mêmes lettres marquent les mêmes chofes que dans la Fig. précedente) perpendiculairement en $O$ fur $A O \mathrm{la}$ droite $O \mathscr{Q}=\frac{\mathrm{aag} ;}{\mathrm{a} \varepsilon \mathrm{c}}$; $\&$ du centre 2 entreles afymptores $20,2 R$, une hyperbole équilatere $V X Z$, dont le rectangle (descoordonnées) 2 YXou QOZ $a a$; foic prolongée une ordonnée quelconque $x x$ de l'hyperbole jufqu'à ce qu'elle rencontre le cercle $M S T$ en $S$, par lequel point $\dot{S}$ foir
menée $O S$, fur laquelle

(prolongée 's'il en eft befoin) foit prife $O B=X Y$. Je dis que le point $B$ fera un de ceux de la rrajectoire $A B C$; puifque

$$
O B=\frac{O Q \cdot O A}{O Q-O Y} .
$$

Substituting in this expression $O Y=O T \sin (\theta), \epsilon=O T / O Q=h\left(2 c^{2} / a^{2} g\right)$ and $O A=2 c^{2} / g$, shows that Bernoulli's geometric construction ${ }^{42}$ for the curve $A B C$ corresponds to Eq. 64 .

In his next step, Bernoulli gave an algebraic proof that the curve $A B C$, determined geometrically in polar coordinates $r, \theta$, satisfies the algebraic equation for a conic section, in cartesian coordinates, ${ }^{43} x=O F$ and $y=F B$, where $F$ is the intersection of the vertical line from $B$, with the horizontal line $O Q$. In his diagram, Fig. 5, the origin of coordinates at $O$ is one of the foci of the conic, and

$$
\begin{aligned}
& x=\frac{O B \cdot O Y}{O T} \\
& y=\frac{O B \cdot S Y}{O T}
\end{aligned}
$$

where $O B=a^{2} /\left(a^{2} g / 2 c^{2}-t\right)$, Eq. 61, $O Y / O T=t / h$ and $S Y / O T=$ $\sqrt{\left(h^{2}-t^{2}\right)} / h$.

After this geometrical considerations, Bernoulli obtained an algebraic equation for $x, y$ by eliminating the variable $t$ which appears in Eqs. 70 and 71, but he did not describe his derivation which is, however, easy to reconstruct.

\footnotetext{
42 Newton, who expressed his propositions and solutions in the Principia in geometrically form, could have used Bernoulli's construction in the second and third edition of his Principia to include the famous "missing" corollary of Prop. 41 for inverse square forces. Most likely, however, he failed to do this because his animosity towards Bernoulli, which also led to his refusal to give him any credit for this achievement. 43 We follow here Bernoulli who used the same notation for the radial coordinate, and for its cartesian component along the $x$-axis.
} 
Setting $\eta=a^{2} g / 2 c^{2}$, Eq. 70 for $x$ yields

$$
t=\frac{\eta h x}{h x+a^{2}}
$$

and substituting this expression for $t$ in Eq. 71 for $y$ gives

$$
\left(\eta^{2}-h^{2}\right) x^{2}=2 a^{2} h x-\eta^{2} y^{2}+a^{4},
$$

where $h / \eta=\epsilon$ is the eccentricy parameter. ${ }^{44}$ In this manner, Bernoulli gave an algebraic proof that his geometrical curve $A B C$ corresponds to the curve for a conic section, Eq. 64, which he wrote in the corresponding form

$$
\left(a^{4} g^{2}-4 c^{4} h^{2}\right) x^{2}=8 a^{2} c^{4} h x-a^{4} g^{2} y^{2}+4 a^{4} c^{4} .
$$

In reference to Bernoulli's translation of Props. 39-41 in the Principia into the notation introduced by Leibniz, Keill perceptively observed that

The solution of M.Bernoully does not differ from that of M.Newton except in the characters or symbols...it will result that that formula does not differ from the Newtonian more than the same words when written in Latin and in Greek (Guicciardini 2008)

Keill's judgement hits the nail on its head. ${ }^{45}$ Like Newton, Bernoulli was well versed in both geometrical and algebraic techniques, and he employed both approaches to achieve his result.

\section{Concluding remarks}

In the late seventeenth century, the analytic calculus of Newton and Leibniz introduced finite differentials which were grounded on geometrical quantities readily comprehensible to its practitiones. The difficulty associated with the mathematical limit - that at the end of a calculation the differentials, like ghosts, had to vanish-was not resolved rigorously until a century later, but this problem was left to be debated as a philosophical problem of mathematics; it did not delay progress in the development and application of the new calculus. Later on, after Clairaut, d'Alembert and Euler formalized the calculus into the form familiar to us today, all traces of the geometrical origin

\footnotetext{
44 Setting $h / \eta=c$, Bernoulli's algebraic equation corresponds to Herman's result, Eq. 16, with $a$ substituted by $a / \sqrt{\eta}$.

45 Although Guicciardini agrees that Bernoulli's expression, Eq. 58, is “a translation of Newton's geometrical proportionalities into the language of calculus", he questions whether Newton could have performed such a translation, claiming that "he used his own method of series and fluxions that is equivalent and yet different from Leibniz's algorithm" (Guicciardini 2008). Yet the explanation that Newton gave to David Gregory on how he obtained the solution for the inverse cube force, described in Cor. 3 of Prop. 41, shows that Newton's procedures were virtually identical to those of Bernoulli.
} 
Fig. 6 Newton's diagram for Lemma 2, sect 1 of the Principia

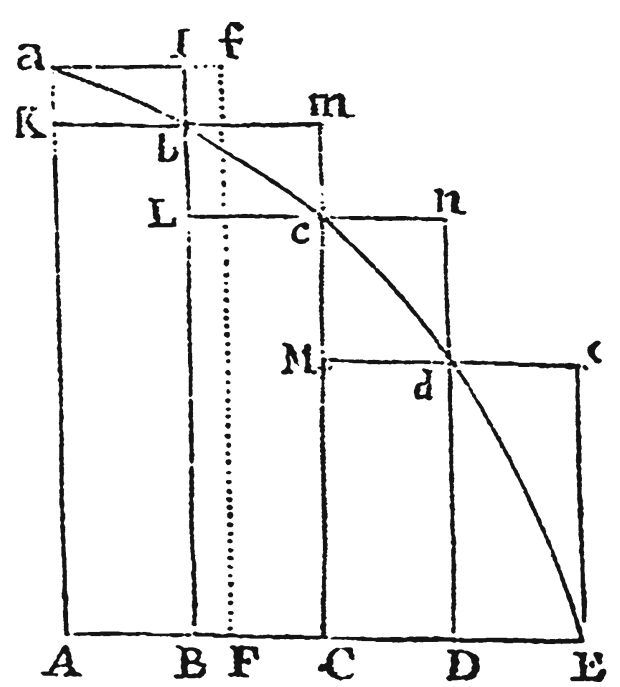

of these equation were removed. Leibniz foresaw this development with his remark that

In truth no one before Leibniz has had the idea of constituting out of this new calculus and algorithm whereby the imagination would be freed from the perpetual attention to figures (Leibniz 1849)

In a letter to Christiann Huygens, he wrote

For what I love most about my calculus is that it gives us the same advantages over the Ancients in the geometry of Archimedes, that Viète and Descartes have given us in the geometry of Euclid or Apollonios, in freeing us from having to work with the imagination (Huygens 1905)

Leibniz's remarks offers some insight why the connection between mathematics and reality has become a riddle in modern times. The standard abstract formulation of Newtonian mechanics based on the equation $f=m a$, where the acceleration $a$ is represented by a second derivative, hides its geometric origins. Indeed, it is the "attention to figures", which Newton advocated, that give us some understanding of the connection of this differential equation to reality. We have shown that in the work of Bernoulli, Hermann, Leibniz and Varignon, the problem of inverse square forces was first visualized geometrically, and then solved analytically with finite differentials that satisfy algebraic equations.

\section{Appendix 1. Early formulation of the fundamental theorem of the calculus}

In Prop. X:11 of his 1668-1669 Geometrical Lectures (Barrow 1916; Mahoney 1990), Isaac Barrow gave a rigorous geometrical proof of the fundamental theorem of 
the calculus. ${ }^{46}$ Barrow proved that the subtangent of a curve with ordinate $Y_{x}$ for the area bounded by another curve $y_{x}$ at a corresponding value of $x$ of the abscissa, is proportional to the ratio $Y_{x} / y_{x}$. In a 1693 article in the Acta Eruditorum (Leibniz 1693), Leibniz essentially took over Barrow's geometrical proof, substituting the differential form for the subtangent, $Y_{x} \mathrm{~d} x / \mathrm{d} Y_{x}$, to write Barrow's result, $Y_{x} \mathrm{~d} x / \mathrm{d} Y_{x}=a Y_{x} / y_{x}$, in the form $a Y_{x}=\int y_{x} \mathrm{~d} x$, where $a$ is an arbitray constant of proportionality with the dimension of length.

In an appendix to his lectures, Barrow also gave a bound for the area of a curve by the sum of the areas of rectangles, which later Newton adopted as Lemma 2 of Sect. 1 in the Principia. Barrow's bound served as the starting point for an analytic proof of the fundamental theorem along the lines developed by Leibniz and Newton. Referring to Fig. 6, which corresponds to Barrow's diagram as it appeared in Lemma 2, let $y_{i}$ be the ordinate of the curve at $x_{i}$ for $i=1-5$. Then $y_{1}=A a, y_{2}=B b, y_{3}=C d, y_{4}=D d$ and $y_{5}=E e$, and $x_{1}=0, x_{2}=A B, x_{3}=A C, x_{4}=A D$, and $x_{5}=A E$, where $A B=B C=C D=D E$, i.e., the corresponding rectangles have equal differential widths $\mathrm{d} x=x_{i+1}-x_{i}$. The upper bound $U$ for the area under the curve is

$$
U=\sum_{i=1}^{i=4} y_{i} \mathrm{~d} x,
$$

the lower bound $D$ is

$$
D=\sum_{i=2}^{i=4} y_{i} \mathrm{~d} x,
$$

and the difference $U-D$ is the area of the first rectangle

$$
U-D=y_{1} \mathrm{~d} x
$$

Geometrically, this result can also be seen to be the sum of the area of the residual rectangles $a I b K, b m c L, c n d M$ and deED. Of course, this result applies also for a subdivision with $n$ equal rectangles. It follows that when the magnitude of the differential $\mathrm{d} x$ is decreased, and accordingly the number $n$ of rectangles is increased, the difference $U-D$ between the upper and lower bounds to the area under the curve, which is proportional to $\mathrm{d} x$, also decreases. In the limit that $\mathrm{d} x$ becomes vanishingly small, this difference becomes zero giving a proof equivalent to Archimedes method by exhaustion that either bound approaches the area.

Hence, by taking a sufficiently large number $n$ of rectangles, the sum of differentials

$$
Y_{n}=\sum_{i=1}^{i=n} y_{i} \mathrm{~d} x
$$

\footnotetext{
46 In a letter to Oldenburg meant for Leibniz, written on Oct 24,1676 , Newton formulated this theorem in the form of an anagram that reads "given an equation involving any number of fluent quantities to find the fluxions, and conversely" (Newton 1676).
} 
for $i=1$ to $i=n$, where $\mathrm{d} x=x_{i+1}-x_{i}$ is a constant, determines the area under the curve in the interval $x=0, x=x_{n}$ to any desired accuracy. ${ }^{47}$ In turn, the sum $Y_{i}$ satisfies the property that the difference

$$
\mathrm{d} Y_{i}=Y_{i+1}-Y_{i}=y_{i+1} \mathrm{~d} x
$$

is determined by $y_{i+1}$ and the magnitude of the differential $\mathrm{d} x$. Taking $x$ as a variable, let $Y_{x}$ be the area bounded by the curve $y_{x}=y_{i}$, and the ordinates $x_{1}$ and $x$. Then in the limit that $\mathrm{d} x \rightarrow 0$ the ratio $\mathrm{d} Y_{x} / \mathrm{d} x$ approaches the tangent of the curve $Y_{x}$, and according to Eq. 79

$$
\frac{\mathrm{d} Y_{x}}{\mathrm{~d} x} \rightarrow y_{x}
$$

This relation represents in algebraic form Barrow's geometrical theorem. In Leibniz's suggestive notation where the summation $\operatorname{sign} \sum$ is replaced by the integral $\operatorname{sign} \int$

$$
Y_{x}=\int_{x_{1}}^{x} y_{x} \mathrm{~d} x
$$

In Newton's equivalent language for the calculus, ${ }^{48}$ the ordinate $y_{x}$ with $x$ as a uniformly increasing variable, i.e. $\dot{x}=1$, represents the fluxion associated with the fluent $Y_{x}$, and the differential relation between $Y_{x}$ and $y_{x}$ is denoted in Newton's notation by a dot on $Y_{x}$,

$$
\dot{Y}_{x}=y_{x}
$$

It is surprising that Newton did not introduce a notation for the integral of $y_{x}$ comparable to Leibniz's, Eq. 81 and instead, he referred to the fluent $Y_{x}$ always as the quadrature or area associated to the curve $y_{x}$. He defined also the moment of any fluent $Y_{x}$ or curve by $\dot{Y}_{x} o$, where $o$ is a small quantity corresponding to Leibniz's differential $\mathrm{d} x$. It is also important to recognize, that Leibniz's differential $\mathrm{d} Y_{x}=Y_{x+\mathrm{d} x}-Y_{x}$ is the same as Newton's moment $\dot{Y}_{x} \mathrm{~d} x$ only to first order in $\mathrm{d} x .^{49}$

\footnotetext{
47 Although the sum $\sum_{i=1}^{i=n} y_{i}$ increases with increasing $n$ without bounds, the product $\left(\sum_{i=1}^{i=n} y_{i}\right) \mathrm{d} x$ remains finite, because $\mathrm{d} x=\left(x-x_{1}\right) / n$ decreases with increasing $n$ in the same proportion.

48 Leibniz's rules for the differential calculus are the same ones that Newton developed for his calculus of fluxions. In 1696, the Marquis De L'Hospital published Analyse des Infiniment Petits pour L'Intelligence des Lignes Courbes, based on lectures that he had received from Bernoulli, which became the first textbook on the differential calculus in the Continent. It was translated from French to English in 1730 by E. Stone under the title The Method of Fluxions both Direct and Inverse. Stone changed Leibniz differential notation into Newton's dot notation for fluxions, e.g., $\mathrm{d} x$ became $\dot{x} \mathrm{~d} t$, and he added his own description of the integral calculus.

49 The ratio $\mathrm{d} Y^{\prime} / \mathrm{d} x$ of Leibniz's "characteristic triangle" with sides $\mathrm{d} x$ and $\mathrm{d} Y^{\prime}$ is equal to the tangent $\dot{Y}$ at $x$. Leibniz used the same symbol $\mathrm{d} Y$ for this different definition of the differential $\mathrm{d} Y^{\prime}$, which for a fixed value of $\mathrm{d} x$ is equal to Newton's definition of the moment of Y.
} 
While a rigorous mathematical formulation of the limit $\mathrm{d} x \rightarrow 0$ had to wait for another century, in Lemma1, Sect. 1 of the Principia Newton expressed this limit concisely under the heading of "first and ultimate ratios" as follows:

Quantities and also ratios of quantities, which in any finite time constantly tend to equality, and which before the end of that time approach so close to one another that their difference is less than any given quantity, become ultimately equal.

In practice, differentials, either geometrical or algebraic, were always represented by finite albeit very small quantities. Both Newton and Leibniz recognized that for sufficiently small values of these differentials, the results obtained from approximate relations in which the contributions of higher order differentials were neglected would be accurate to any desired degree.

\section{Appendix 2}

Using vector notation with Cartesian components along the $x, y$ axis and origin at the center of force, we give a succinct derivation of the second-order differentials $\mathrm{dd} x$ and $\mathrm{dd} y$ for the force impulse along the lines given by Hermann based on Prop. 1 in Newton's. This derivation is contrasted with Bernoulli's, which was given in polar coordinates, and with by a comparable derivation based on Prop. 6 .

In reference to Hermann's diagram shown in Fig. 2, let the positions $B, C$ and $E$ on the curve $A B C D$. be represented by the vectors $\vec{r}_{B}=\vec{r}(t), \vec{r}_{C}=\vec{r}(t+\mathrm{d} t)$, and $\vec{r}_{D}=\vec{r}(t+2 \mathrm{~d} t)$. Then $\vec{r}_{E}=2 \vec{r}_{C}-\vec{r}_{B}$, and $\overrightarrow{E D}=\vec{r}_{D}-\vec{r}_{E}$ is given by

$$
\overrightarrow{E D}=\vec{r}(t+2 \mathrm{~d} t)+\vec{r}(t)-2 \vec{r}(t+\mathrm{d} t))
$$

Defining the first-order differential by $\overrightarrow{\mathrm{d}} r(t)=\vec{r}(t+\mathrm{d} t)-\vec{r}(t)$, and the second-order differential by $\overrightarrow{\mathrm{d} d} r(t)=\overrightarrow{\mathrm{d}} r(t+\mathrm{d} t)-\overrightarrow{\mathrm{d}} r(t)$, we find that

$$
\overrightarrow{E D}=\overrightarrow{\mathrm{d}} \mathrm{d} r(t)
$$

For an attractive central inverse square force $\vec{E} D=E D \vec{r} / r$, where $r=\sqrt{x^{2}+y^{2}}$, $E D=-(x \mathrm{~d} y-y \mathrm{~d} x)^{2} / a r^{2}$, and we obtain

$$
d d \vec{r}=-\frac{\vec{r}}{r^{3}}(x \mathrm{~d} y-y \mathrm{~d} x)^{2} .
$$

which corresponds to Hermann's result, Eq. 4 , for the $x$ component of $\vec{r}$.

In polar coordinates $r, \theta$, one also must take into account that the reference unit vectors $\vec{u}, \vec{v}$ along the radial and transverse direction are not fixed in space, but satisfy the conditions $d \vec{u}=\vec{v} d \theta$, and $d \vec{v}=\vec{u} d \theta$. Setting $\vec{r}=r \vec{u}$, we have

$$
d \vec{r}=\mathrm{d} r \vec{u}+r \mathrm{~d} \theta \vec{v}
$$


and

$$
d d \vec{r}=\left(\mathrm{d} d r-r \mathrm{~d} \theta^{2}\right) \vec{u}+(r d d \theta+2 \mathrm{~d} r \mathrm{~d} \theta) \vec{v} .
$$

For a force impulse along the radial direction, $\xi \vec{u}$, the second-order differential component of $\overrightarrow{\mathrm{d} d} r$ along the transverse direction $\vec{v}$ must vanish, leading to Kepler's area law in the form

$$
\mathrm{d}\left(r^{2} \mathrm{~d} \theta\right)=0
$$

Then

$$
\xi \propto \mathrm{dd} r-r \mathrm{~d} \theta^{2}
$$

which is the form originally obtained by Leibniz and re-derived by Bernoulli. Setting $\xi=-2 \phi c^{2}$, where $\phi$ is the force and $c=r^{2} \mathrm{~d} \theta$ is a constant, we recover Bernoulli's result

$$
\phi=-\frac{\left(\mathrm{d} d r-r \mathrm{~d} \theta^{2}\right)}{2 c^{2}}
$$

In the diagram associated with Prop. 6, shown in Fig. 4, let $\vec{r}_{P}=\vec{r}(t), \vec{r}_{Q}=$ $\vec{r}(t+\mathrm{d} t))$ represent the positions $Q$ and $P$ on the curve, and $\vec{r}_{R}(t)=\vec{r}(t)+\vec{v}(t) \mathrm{d} t$, the position of $R$, where $\vec{v}(t)$ is the velocity at $P$ along the tangent line $R P$. Hence the change in position $\vec{R} Q=\vec{r}_{R}-\vec{r}_{Q}$, is

$$
\overrightarrow{R Q}=-\overrightarrow{\mathrm{d}} r(t)+\vec{v}(t) \mathrm{d} t
$$

To convert this expression to differentials, we need to express the velocity vector $\vec{v}(t)$ in terms of first-order differentials $\overrightarrow{\mathrm{d}} r(t)$ with the property that in the limit when $Q$ approaches $P$, this vector remains tangential to the curve at $P$ up to third order in powers of $\mathrm{d} t$. Introducing $Q^{\prime}$ for the position on the orbit at time $t-\mathrm{d} t$, which is not shown in Newton' diagram, where $\vec{r}_{Q^{\prime}}=\vec{r}(t-\mathrm{d} t)$, this property is satisfied uniquely by the chord $\vec{r}_{Q}-\vec{r}_{Q^{\prime}}=\overrightarrow{\mathrm{d}} r(t)+\overrightarrow{\mathrm{d}} r(t-\mathrm{d} t)$. Hence, we approximate $\vec{v}(t)$ by the relation

$$
\vec{v}(t) \mathrm{d} t=\frac{1}{2}(\overrightarrow{\mathrm{d}} r(t)+\overrightarrow{\mathrm{d}} r(t-\mathrm{d} t))
$$

and substituting this expression for $\vec{v}(t) \mathrm{d} t$ in Eq. 91, we obtain

$$
\overrightarrow{R Q}=\frac{1}{2} \overrightarrow{\mathrm{d}} \mathrm{d} r(t)
$$

Acknowledgements I would like to thank Niccolo Guicciardini for numerous discussion and many helpful comments and corrections of an earlier draft of this manuscript. 
Open Access This article is distributed under the terms of the Creative Commons Attribution Noncommercial License which permits any noncommercial use, distribution, and reproduction in any medium, provided the original author(s) and source are credited.

\section{References}

Aiton, E. J., 1954 The Inverse Problem of Central Forces. Annals of Science 20:81-99

Aiton, E. J., 1962 The Celestial Mechanics of Leibniz. Annals of Science 16:65-82

Aiton, E. J., 1995 The vortex theory in competition with Newtonian celestial dynamics. Planetary astronomy from the Renaissance to the rise of astrophysics, part B, edited by R. Taton and C. Wilson (Cambridge University Press) pp. 3-21

Barrow, I., 1916 The Geometrical Lectures of Isaac Barrow. Translations and notes by J. M. Child (Open Court, London), Quoted by N. Guicciardini in reference (Guicciardini 2009)

Bernoulli, J., 1710 Extrait de la Résponse de M. Bernoulli à M. Herman, datée de Basle le 7. Octobre 1710. Memoires de l'Academie Royale des Sciences pp. 521-533

Bernoulli, J., 1914 Die erste Integralrechnungen Ostwald Klassiker nr. 194 (Leipzig und Berlin, 1914) 63-67. Archives for the History of Exact Sciences 46, 222-251

Bernoulli, J., 1999 Die Werke von Jakob Bernoulli: Bd. 5: Differentialgeometrie edited by D. Speiser, A. Weil and M. Mattmüller (Birkhaüsser)

Bernoulli, 2008 Die Werke von Johannn I und Nicolaus II Bernoulli ed. P. Radelet-de Grave (Birkhäuser) pp. 125-128

Bertoloni Meli, D., 1991 Equivalence and priority: Newton vs. Leibniz (Clarendon Press, Oxford)

Boyer, C. B., 1989 A History of Mathematics (Wiley, New York)

Bos, H. J. M., 1973 Differentials, Higher-Order Differentials and the Derivative in the Leibnizian Calculus. Arch. Hist. Exact Sci. 14:1-90

Brackenridge, J. B., 2003 Newton's Easy Quadratures “Omitted for the Sake of Brevity". Archive for History of Exact Sciences 57, 313-336

Cohen, I. B., 1999 Isaac Newton The Principia. A new translation by I. B. Cohen and Anne Whitman assisted by Julia Budenz, and A Guide to Newton's Principia by I. B. Cohen (University of California Press) p. 115

Erlichson, H., 1994 The Visualization of Quadratures in the Mystery of Corollary 3 to Proposition 41 of Newton's Principia. Historia Mathematica 21, 148-161

Euler, L., 1736 Mechanica (St. Petersburg) Quoted in reference (Bertoloni Meli 1991) p. 214

Guicciardini, N., 1999 Reading the Principia: The Debate on Newton's Mathematical Methods for Natural Philosophy from 187 to 1736 . (Cambridge University Press, Cambridge) pp. 207-225

Guicciardini, N., 2008 Isaac Newton and Johann Bernoulli on central force motion. (to be published)

Guicciardini, N., 2009 Isaac Newton on Mathematical Certainty and Method (M.I.T. Press, Cambridge)

Hermann, J., 1710a Extrait d'une Lettre de M. Herman à M. Bernoulli, datée de Padoüe le 12. Juillet 1710. Memoires de l'Academie Royale des Sciences pp. 519-521

Hermann, J., 1710b Metodo d'investigare l'Orbite de' Pianeti...Giornale de' Letterati d' Italia, vol. 2, $447-467$

Huygens, C., 1673 Horologium Oscillatorium (Paris). Reproduced in Ouvres Complètes de Christiaan Huygens, vol. 8 (Martinus Nijhoff). English translation in Richard J. Blackwell, Christiaan Huygens's Pedulum Clock (Ames 1986)

Huygens, C., 1905 Ouvres Complètes des Christiaan Huygens vol. 10 Correspondance 1691-1695 (Martinus Nijhoff, The Hague) p. 227. Translation from French to English by N. Guicciardini in ref. (Guicciardini 1999) p. 166

Kline, M., 1972 Mathematical Thought from Ancient to Modern Times, vol. 1 (Oxford University Press, Oxford)

Laplace, P. S., 1798 Traité de Méchanique Celeste Tome I, Premier Partie, Livre II, (Gauthier-Villars, Paris) p. 165, Ouvres Complétes de Laplace (Gauthiers-Villars, Paris, 1843) p. 181

Leibniz, G., 1693 Supplementum geometriae dimensoriare ... Acta Eruditorum, 385-392. Translated into English from Leibniz, Mathematische Schriften, Abth. 2, Band I, 294-301, in A source book in mathematics, edited by E.D. Struik (Harvard University Press 1969) pp. 282-284

Leibniz, G. W., 1849 Mathematische Schriften vol. 5, ed. C. I. Gerhardt (Berlin) p. 393 
Leibniz, G. W., 1973 Marginalia in Newoni Prncipia Mathematica (1687) edited by E. A. Fellman (Librarie Philosophique J Vrin, Paris)

Mahoney, M. S., 1990 Barrow's mathematics: between ancients and moderns, in Before Newton: The life and times of Isaac Barrow, edited by Mordechai Feingold (Cambridge University Press, Cambridge) pp. 179-249

Mazzone, S. and Roero, C. S., 1997 Jacob Hermann and the diffusion of the Leibnizian calculus in Itally (Olschki, Firenze)

Nauenberg, M., 1994 Newton's Early Computation for Dynamics

Nauenberg, M., 2003 Kepler's area law in the Principia: filling in some details in Newton's proof of Proposition 1. Historia Mathematica 30:441-456

Nauenberg, M., 2005 Robert Hooke's seminal contributions to orbital dynamics. Physics in Perspective 7, 4-34; reprinted in Robert Hooke Tercentennial Studies, edited by M. Cooper and M. Hunter (Ashgate, Cornwall, 2006) Physics in Perspective

Newton, I., 1670-1673 The Mathematical Papers of Isaac Newton vol. 3, 1670-1673, edited by D.T. Whiteside (Cambridge University Press) pp. 154-155

Newton, I., 1960 The correspondence of Isaac Newton vol. 3 edited by H. W. Turnbull (Cambridge University Press) p. 297

Newton, I., 1968 The Mathematical Papers of Isaac Newton vol. 2, edited by D.T. Whiteside (Cambridge Universit Press, Cambridge) p. 270

Newton, I., 1974 The Mathematical Papers of Isaac Newton vol. 6, 1684-1691, edited by D.T. Whiteside (Cambridge University Press, 1974) pp. 35-37

Newton, I., 1676 The correspondence of Isaac Newton, vol. 2, 1676-1687, edited by H.W. Turnbull (Cambridge University Press 1976)

Varignon, M., 1710 Des forces Centrales Memoires de l'Academie Royale des Sciences 533-544

Wilson, C., 1994 Newton's Orbit Problem: A Historian's Response. The College Mathematics Journal 25:193-205 